Canadian

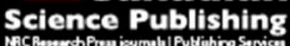

Applied Physiology, Nutrition, and Metabolism Physiologie appliquée, nutrition et métabolisme

\title{
Physiological and Perceptual Responses to Incremental Exercise Testing in Healthy Men: Effect of Exercise Test Modality
}

\begin{tabular}{|r|l|}
\hline Journal: & Applied Physiology, Nutrition, and Metabolism \\
\hline Manuscript ID & apnm-2015-0179.R1 \\
\hline Manuscript Type: & Article \\
\hline Date Submitted by the Author: & $19-J u l-2015$ \\
\hline Complete List of Authors: & $\begin{array}{l}\text { Muscat, Kristina; McGill University, Kinesiology \& Physical Education } \\
\text { Kotrach, Houssam; McGill University, Kinesiology \& Physical Education } \\
\text { Wilkinson-Maitland, Courtney; McGill University, Kinesiology \& Physical } \\
\text { Education } \\
\text { Schaeffer, Michele; McGill University, Kinesiology \& Physical Education } \\
\text { Mendonca, Cassandra; McGill University, Kinesiology \& Physical Education } \\
\text { Jensen, Dennis; McGill University, Kinesiology \& Physical Education }\end{array}$ \\
\hline Keyword: & $\begin{array}{l}\text { exercise testing < exercise, aerobic evaluation < exercise, exercise } \\
\text { physiology < exercise, exercise prescription < exercise, physiological } \\
\text { assessment of performance < physiological assessment of performance }\end{array}$ \\
\hline \multicolumn{2}{|l}{} \\
\hline
\end{tabular}


1 Physiological and Perceptual Responses to Incremental Exercise Testing in Healthy Men: Effect of Exercise Test Modality

Kristina M. MUSCAT ${ }^{1}$ (kristina.muscat@mail.mcgill.ca)

Houssam G. KOTRACH ${ }^{1}$ (hussam.kotrach@mail.mcgill.ca)

Courtney A. WILKINSON-MAITLAND ${ }^{1}$ (courtney.wilkinson-maitland@,mail.mcgill.ca)

8 Michele R. SCHAEFFER ${ }^{1}$ (michelerschaeffer@gmail.com)

9 Cassandra T. MENDONCA ${ }^{1}$ (ct.mendonca@gmail.com)

10 Dennis JENSEN ${ }^{1,2,3}$ (dennis.jensen@,mcgill.ca)

${ }^{1}$ Clinical Exercise \& Respiratory Physiology Laboratory, Department of Kinesiology \& Physical

${ }^{2}$ Respiratory Epidemiology and Clinical Research Unit, Montréal Chest Institute, McGill

16 University Health Centre, Montréal, QC, Canada.

${ }^{3}$ Research Centre for Physical Activity and Health, McGill University, Montréal, QC, Canada.

Running Head: Exercise responses to treadmill vs. cycle ergometry

\section{Corresponding Author:}

Dennis Jensen, Ph.D.

Department of Kinesiology and Physical Education

475 Pine Avenue West

Montréal, QC, Canada

27 H2W 1S4

28 Phone: (514) 398-4184

29 Fax: (514) 398-4186

30 Email: dennis.jensen@mcgill.ca 


\section{ABSTRACT}

34 In a randomized cross-over study of 15 healthy men aged 20-30 years, we compared

35 physiological and perceptual responses during treadmill and cycle exercise test protocols

36 matched for increments in work rate - the source of increased locomotor muscle metabolic and

37 contractile demands. The rates of $\mathrm{O}_{2}$ consumption $\left(\dot{\mathrm{VO}}_{2}\right)$ and $\mathrm{CO}_{2}$ production $\left(\dot{\mathrm{VCO}}_{2}\right)$ were

38 higher at the peak of treadmill vs. cycle testing $(p \leq 0.05)$. Nevertheless, work rate, minute

39 ventilation $\left(\dot{\mathrm{V}}_{\mathrm{E}}\right)$, tidal volume $\left(\mathrm{V}_{\mathrm{T}}\right)$, breathing frequency $\left(f_{\mathrm{R}}\right)$, inspiratory capacity (IC),

40 inspiratory reserve volume (IRV), tidal esophageal (Pes,tidal) and transdiaphragmatic pressure

41 swings (Pdi,tidal), peak expiratory gastric pressures (Pga,peak), the root mean square of the

42 diaphragm EMG (EMGdi,rms) expressed as a percentage of maximum EMGdi,rms

43 (EMGdi,rms\%max), and dyspnea ratings were similar at the peak of treadmill vs. cycle testing

44 ( $>0.05)$. Ratings of leg discomfort were higher at the peak of cycle vs. treadmill exercise

$45(\mathrm{p} \leq 0.05)$, even though peak $\dot{\mathrm{V}} \mathrm{O}_{2}$ was lower during cycling. $\dot{\mathrm{V}} \mathrm{O}_{2}, \dot{\mathrm{V}} \mathrm{CO}_{2}, \dot{\mathrm{V}}_{\mathrm{E}}, f_{\mathrm{R}}$, Pes,tidal,

46 Pdi,tidal and Pga,peak were higher $(\mathrm{p} \leq 0.05)$, while $\mathrm{V}_{\mathrm{T}}, \mathrm{IC}, \mathrm{IRV}$, EMGdi,rms\%max, and ratings

47 of dyspnea and leg discomfort were similar $(\mathrm{p}>0.05)$ at all or most submaximal work rates during

48 treadmill vs. cycle exercise. Our findings highlight important differences (and similarities) in

49 physiological and perceptual responses at maximal and submaximal work rates during

50 incremental treadmill and cycle exercise testing protocols. The lack of effect of exercise test

51 modality on peak work rate advocates for the use of this readily available parameter to optimize

52 training intensity determination, regardless of exercise training mode.

53 Key Words: Exercise; Bicycle; Treadmill; Mode; Symptom; Physiology 


\section{INTRODUCTION}

55 The two most commonly used modes of exercise in research and clinical settings are the

56 treadmill and cycle ergometer (American Thoracic and American College of Chest 2003). In the

57 exercise training and/or rehabilitation setting, it is generally accepted that most individuals prefer

58 to exercise on a treadmill than on a cycle ergometer. Indeed, Cumming (1977) reported that $73 \%$

59 of 305 normal males aged $42-70$ yrs indicated preference for treadmill walking/running over

60 cycle ergometry in part because it was felt to be less fatiguing on the legs. Over the past 45

61 years, many studies have sought to identify differences (and similarities) in the acute

62 physiological response to treadmill and cycle ergometer exercise testing. The best-known

63 difference between these modalities is in the maximal rate of $\mathrm{O}_{2}$ consumption $\left(\mathrm{V}_{2 \max }\right)$, which is

64 up to $20 \%$ higher when exercise testing is performed on a treadmill vs. cycle ergometer in both

65 health and disease (Achten et al. 2003; Ciavaglia et al. 2014b; Faulkner et al. 1971; Hermansen

66 et al. 1970; Hermansen and Saltin 1969; Jacobs and Sjodin 1985; Kalsas and Thorsen 2009; Kim

67 et al. 1999; Matsui et al. 1978; Miles et al. 1980; Miyamura and Honda 1972; Okita et al. 1998;

68 Pannier et al. 1980; Porszasz et al. 2003; Tanner et al. 2014). The higher $\dot{\mathrm{V}}_{2 \max }$ response to

69 treadmill vs. cycle exercise testing has been ascribed to several factors, including: recruitment of

70 a relatively larger muscle mass, with attainment of a higher maximal cardiac output,

71 arteriovenous $\mathrm{O}_{2}$ difference, lower limb blood flow and total vascular conductance during

72 treadmill running (Hermansen et al. 1970; Hermansen and Saltin 1969; Kim et al. 1999; Matsui

73 et al. 1978; Miyamura and Honda 1972; Niederberger et al. 1974); higher fat and lower

74 carbohydrate oxidation rates during maximal treadmill vs. cycle ergometry (Achten et al. 2003;

75 Cheneviere et al. 2010); and development of a less severe metabolic acidosis at maximal

76 treadmill vs. cycle exercise (Koyal et al. 1976; Okita et al. 1998). 
While the influence of exercise mode on maximal physiological responses is now well

78 established, only a few studies have examined the influence of modality on physiological and

79 perceptual responses at standardized submaximal exercise intensities. With few exceptions,

80 these studies have found that carbohydrate oxidation rates, blood lactate concentrations, the

81 respiratory exchange ratio (RER) and the ventilatory equivalent for $\mathrm{O}_{2}\left(\dot{\mathrm{V}}_{\mathrm{E}} / \dot{\mathrm{V}}_{2}\right)$ are lower,

82 while intensity ratings of breathing and leg discomfort are not different at equivalent submaximal

83 exercise intensities during treadmill vs. cycle ergometry (Duke et al. 2014; Hermansen et al.

84 1970; Hermansen and Saltin 1969; Jacobs and Sjodin 1985; Koyal et al. 1976; Miles et al. 1980;

85 Miyamura and Honda 1972; Sharma et al. 2015). In each of these studies, however, measured

86 parameters were compared between modalities at equivalent submaximal levels of $\dot{\mathrm{V}} \mathrm{O}_{2}$,

87 expressed either in $\mathrm{Lmin}^{-1}, \mathrm{mLkg}^{-1} \mathrm{~min}^{-1}$ or as a percentage of $\dot{\mathrm{V}} \mathrm{O}_{2 \max }$. As such, the true impact

88 of test modality on exercise physiological and perceptual responses is difficult to interpret from

89 these earlier studies in as much as the protocols were not matched for increments in external

90 work rate, which is the proximate source of increased skeletal (locomotor) muscle metabolic and

91 contractile demands.

92 In 2003, Porszasz et al. (2003) first described an incremental treadmill protocol that

93 utilizes linear increases in speed and nonlinear increases in grade that result in ramp-like

94 increments in external work rate equivalent to those produced by an electronically-braked cycle

95 ergometer. In this study of 22 healthy, sedentary adults, the slope of the $\mathrm{VO}_{2}$-work rate

96 relationship was linear and significantly higher (by 19\%) during incremental treadmill vs. cycle

97 exercise. Apart from $\dot{\mathrm{VO}}_{2}$, however, Porszasz et al. (2003) did not compare cardiovascular, gas

98 exchange, breathing pattern, operating lung volume, respiratory mechanical/muscular and/or

99 perceptual responses at equivalent submaximal work rates between modalities. 
Ciavaglia et al. (2014a; 2014b) recently compared a constellation of detailed

101 physiological and perceptual responses to symptom-limited treadmill and cycle exercise using

102 matched incremental work rate protocols in elderly and obese patients with chronic obstructive

103 pulmonary disease (COPD). In these studies, $\dot{\mathrm{V}} \mathrm{O}_{2}$, the rate of $\mathrm{CO}_{2}$ production $\left.(\dot{\mathrm{V} C O})_{2}\right)$ and

104 expiratory muscle activity were higher, while RER, arterial blood $\mathrm{O}_{2}$ saturation and the $\dot{\mathrm{V}}_{\mathrm{E}} / \dot{\mathrm{VO}}_{2}$

105 ratio were lower at any submaximal work rate during treadmill vs. cycle ergometry. Despite

106 these differences, mean values for heart rate (HR), the ventilatory equivalent for $\mathrm{CO}_{2}$

$107\left(\dot{\mathrm{V}}_{\mathrm{E}} / \dot{\mathrm{V} C O}_{2}\right)$, minute ventilation $\left(\dot{\mathrm{V}}_{\mathrm{E}}\right)$, tidal volume $\left(\mathrm{V}_{\mathrm{T}}\right)$, breathing frequency $\left(f_{\mathrm{R}}\right)$, inspiratory

108 capacity (IC), inspiratory reserve volume (IRV), neural inspiratory drive, and intensity ratings of

109 dyspnea and leg discomfort were similar at any equivalent submaximal work rate during

110 treadmill vs. cycle exercise. It is unlikely that these novel and important observations from

111 elderly and obese COPD patients can be generalized to healthy, young and non-obese adults.

112 Accordingly, our study is the first to compare detailed assessments of cardio-metabolic

113 function, gas exchange, breathing pattern, dynamic operating lung volumes, neural inspiratory

114 drive, inspiratory and expiratory muscle function, dyspnea and leg discomfort in healthy, young

115 and non-obese adults during symptom-limited treadmill and cycle exercise test protocols

116 carefully matched for increments in work rate.

\section{METHODS}

119 Participants. Participants included healthy, non-smoking, non-obese (Body mass index (BMI)

$120<30 \mathrm{kgm}^{-2}$ ) men aged 20-30 yrs with normal spirometry (Vestbo et al. 2013). Participants were

121 excluded if they had a known or suspected history of cardiovascular, respiratory,

122 musculoskeletal, endocrine, neuromuscular and/or metabolic disease; were taking doctor 
123 prescribed medications; and/or had an allergy to lidocaine. Participants were recruited from the

124 Montréal and surrounding area by word-of-mouth and online postings in the McGill University

125 and Concordia University classifieds.

127 Study Design. Participants visited the Clinical Exercise and Respiratory Physiology Laboratory

128 at McGill University on 3 separate occasions. Visit 1 included screening for eligibility criteria,

129 spirometry, and a symptom-limited incremental cycle exercise test followed 45-min thereafter by

130 a symptom-limited incremental treadmill exercise test (both for familiarization purposes). Visits

1312 and 3 included spirometry and a symptom-limited incremental cycle or treadmill exercise test

132 (randomized visit order) with added measurements of the diaphragm electromyogram and of

133 respiratory pressures. Participants were instructed to avoid alcohol, caffeine and strenuous

134 exercise on each test day, which were separated by $\geq 48$-hr and conducted at the same time of day

135 ( $\pm 1 \mathrm{hr}$ ) for each participant. The study protocol and consent form were approved by the

136 Institutional Review Board of the Faculty of Medicine at McGill University (A00-M74-11B) in

137 accordance with the Declaration of Helsinki. All participants provided written informed consent.

139 Pulmonary function tests. Forced vital capacity (FVC), forced expiratory volume in 1-sec

$140\left(\mathrm{FEV}_{1}\right)$ and the $\mathrm{FEV}_{1} / \mathrm{FVC}$ ratio were determined using automated testing equipment (Vmax

141 Encore ${ }^{\mathrm{TM}}$; CareFusion, Yorba Linda, CA, USA). Measurements were obtained with participants

142 seated, utilizing recommended techniques (Miller et al. 2005) and expressed as a percentage of

143 predicted normal values (Hankinson et al. 1999). 
145 Cardiopulmonary exercise testing. Incremental exercise tests were conducted on either an

146 electronically braked cycle ergometer (Ergoline 800s; CareFusion, Yorba Linda, CA, USA) or a

147 motorized treadmill (Trackmaster Model \#TMX425C; Newton, KS, USA) using a Vmax

148 Encore $^{\mathrm{TM}}$ cardiopulmonary exercise testing system; and consisted of a baseline resting period of

$149 \geq 6$-min (while seated on the cycle ergometer and while standing on the treadmill) followed by

150 25-watt increases in work rate (starting at 25 watts) every 2-min to the point of symptom-

151 limitation. The treadmill protocol was individualized for each participant based on their body

152 mass using the formula (Jones 1997): Work rate (watts) $=m^{*} g * v * \sin (\alpha)$, where $m$ is the

153 body mass of the participant in $\mathrm{kg}, g$ is the gravitational acceleration $\left(9.81 \mathrm{~m}^{-1}\right), v$ is the velocity

154 of the treadmill in $\mathrm{ms}^{-1}$, and $\alpha$ is the angle of inclination. The grade started at $5 \%$ and was

155 increased by $1 \%$ every 2 -min, with the increments in treadmill speed required to achieve the 25 -

156 watt $2-\mathrm{min}^{-1}$ increase in work rate determined using the formula. Participants remained seated

157 and maintained a pedaling cadence of $50-100 \mathrm{rev}^{\prime} \mathrm{min}^{-1}$ during cycle testing; and were not

158 allowed to grasp the handrails during treadmill testing.

159 Standard respiratory and gas exchange parameters were collected breath-by-breath at rest

160 and during exercise while participants breathed through a mouthpiece and low-resistance flow

161 transducer with nasal passages occluded by a nose clip. Heart rate was monitored continuously

162 using a Polar ${ }^{\mathbb{B}}$ heart rate monitor (Lachine, QC, Canada). Inspiratory capacity (IC) maneuvers

163 were performed at rest, within the last 30-s of every 2-min stage during exercise, and at end

164 exercise. Assuming that total lung capacity does not change during exercise (Stubbing et al.

165 1980), changes in IC and IRV (calculated as the difference between IC and the simultaneously

166 determined $\mathrm{V}_{\mathrm{T}}$ ) reflect changes in dynamic end-expiratory and end-inspiratory lung volume,

167 respectively. 
169 following questions at rest, within the last 30-s of every 2-min stage during exercise, and at end-

170 exercise: How intense is your sensation of breathing overall? How unpleasant or distressed does

171 your breathing make you feel? How intense is your sensation of leg discomfort? Breathing

172 overall (hereafter referred to as dyspnea) was defined as "the global awareness of your

173 breathing," which is consistent with the American Thoracic Society's most recent

174 recommendation that "...the definition of dyspnea should be neutral with respect to any

175 particular quality" of breathing (Parshall et al. 2012). Prior to each exercise test, participants

176 were familiarized with the Borg scale and its endpoints were anchored such that " 0 " represented

177 "no intensity (unpleasantness) at all" and "10" represented "the most severe intensity

178 (unpleasantness) you have ever experienced or could ever imagine experiencing." At end-

179 exercise, participants verbalized their main reason(s) for stopping exercise (dyspnea, leg

180 discomfort, combination of dyspnea and leg discomfort, other); quantified the percentage

181 contribution of dyspnea and leg discomfort to exercise cessation; and identified qualitative

182 phrases that best described their breathing at end-exercise (O'Donnell et al. 2000).

183 Breath-by-breath measures of the root mean square of the crural diaphragm

184 electromyogram (EMGdi,rms) and of esophageal (Pes), gastric (Pga) and transdiaphragmatic

185 (Pdi, calculated as the difference between Pga and Pes) pressure were recorded from a gastro-

186 esophageal electrode-balloon catheter (Guangzhou Yinghui Medical Equipment Ltd.,

187 Guangzhou, China) and analyzed using published methods (Mendonca et al. 2014; Schaeffer et

188 al. 2014). Maximum voluntary EMGdi,rms (EMGdi,max) was identified as the largest of all

189 EMGdi,rms values obtained from IC maneuvers performed either at rest or during exercise. The

190 ratio of EMGdi,rms to EMGdi,max (EMGdi,rms\%max) was used as an index of neural 
191 inspiratory drive. Tidal swings in Pes (Pes,tidal) and Pdi (Pdi,tidal) were calculated as the

192 difference between peak tidal inspiratory and peak tidal expiratory Pes and Pdi. Peak expiratory

193 Pga (Pga,peak) was taken as an index of expiratory muscle activity (effort).

195 Analysis of exercise end points. Physiological parameters measured breath-by-breath were 196 averaged in 30-s intervals at rest and during exercise. These parameters, collected over the first

197 30-s period of every 2-min stage during exercise, were linked with symptom ratings and IC 198 measurements collected over the latter 30-s of the same minute to avoid contamination of

199 averaged breath-by-breath data by subject-experimenter interaction and by irregular breaths 200 surrounding IC maneuvers.

201 Four main time points were used for the evaluation of exercise parameters: (1) pre202 exercise rest, defined as the average of the last 30 -s of the baseline period after $\geq 2$-min of quiet 203 breathing on the mouthpiece while seated on the cycle ergometer or while standing on the 204 treadmill; (2) ventilatory threshold $\left(\mathrm{T}_{\mathrm{vent}}\right)$, which was identified for each participant and test

205 using the V-slope method (Beaver et al. 1986): physiological parameters and symptom ratings at 206 the $\dot{\mathrm{VO}}_{2}$ corresponding to $\mathrm{T}_{\text {vent }}$ were calculated by linear interpolation between adjacent 207 measurement points for each participant and test; (3) iso-work, defined as the average of the last

20830 -sec of the highest equivalent submaximal work rate achieved by a given participant during

209 both cycle and treadmill tests; and (4) peak exercise, defined as the average of the last 30-s of

210 loaded pedaling and treadmill running. Peak work rate was identified as the highest work rate a

211 given participant was able to sustain for $\geq 30$-s, while exercise endurance time (EET) was defined

212 as the duration of loaded pedaling and as the duration of treadmill walking/running. 
214 Statistical Analysis. The effect of exercise modality, measurement time and their interaction on

215 measured parameters was examined using a two-way repeated measures analysis of variance

216 with correction for multiple comparisons using Tukey's honest significant difference test

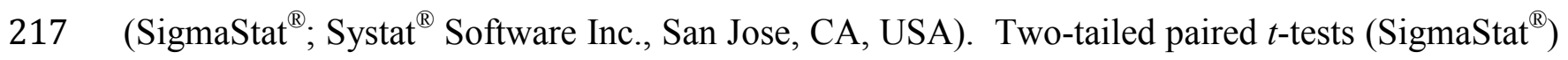

218 were used to examine the effect of exercise mode on 1) measured parameters at $T_{\text {vent }}$ and 2) the

219 percentage contribution of dyspnea and leg discomfort to exercise cessation. Reasons for

220 stopping exercise and qualitative descriptors of dyspnea were assessed using frequency statistics.

221 Significance was set at $\mathrm{p} \leq 0.05$ and data are presented as mean $\pm \mathrm{SEM}$.

\section{RESULTS}

224 Participant characteristics. Participants included 15 healthy, young (22.5 \pm 0.4 yrs), non-obese

$225\left(\mathrm{BMI}=23.8 \pm 0.5 \mathrm{kgm}^{-2}\right)$ men with normal spirometry $\left(\mathrm{FEV}_{1}=107 \pm 3 \%\right.$ predicted; $\mathrm{FEV}_{1} / \mathrm{FVC}$

$226=83 \pm 2 \%$ ) and normal-to-above normal levels of cardiorespiratory: peak $\dot{\mathrm{V}} \mathrm{O}_{2}$ on incremental

227 cycle and treadmill exercise testing of $118 \pm 5$ and $135 \pm 4 \%$ predicted, respectively.

229 Responses to cycle and treadmill exercise. Exercise mode had no effect on EET (cycle, $21.6 \pm$

2300.8 vs. treadmill, $21.4 \pm 0.9 \mathrm{~min}, \mathrm{p}>0.05$ ) or the work rate achieved at peak exercise (cycle, 280

$231 \pm 10$ vs. treadmill, $279 \pm 11$ watts, $\mathrm{p}>0.05)$.

$232 \quad$ Cardiometabolic and gas exchange responses. At the symptom-limited peak of treadmill

233 vs. cycle testing, $\dot{\mathrm{V}} \mathrm{O}_{2}, \dot{\mathrm{V}} \mathrm{CO}_{2}, \mathrm{HR}, \mathrm{O}_{2}$ pulse and $\mathrm{P}_{\mathrm{ET}} \mathrm{CO}_{2}$ were higher, whereas $\mathrm{RER}, \dot{\mathrm{V}} \mathrm{E} / \dot{\mathrm{VO}}_{2}$ and

$234 \mathrm{P}_{\mathrm{ET}} \mathrm{O}_{2}$ were lower (Table 1, Fig. 1).

235 As illustrated in Fig. 1, $\mathrm{VO}_{2}, \dot{\mathrm{V} C O}{ }_{2}, \mathrm{HR}$ and the $\mathrm{O}_{2}$ pulse were higher, while RER was

236 lower at most submaximal work rates during treadmill vs. cycle testing (Table 1). With the 
237 exception of $\dot{\mathrm{V}}_{\mathrm{E}} / \dot{\mathrm{VO}}_{2}$ and $\mathrm{P}_{\mathrm{ET}} \mathrm{O}_{2}$ being higher during cycle vs. treadmill exercise at 25 and 50

238 watts, mean values of $\dot{\mathrm{V}}_{\mathrm{E}} / \dot{\mathrm{VO}}_{2}, \dot{\mathrm{V}}_{\mathrm{E}} / \dot{\mathrm{V}} \mathrm{CO}_{2}, \mathrm{P}_{\mathrm{ET}} \mathrm{O}_{2}$ and $\mathrm{P}_{\mathrm{ET}} \mathrm{CO}_{2}$ were similar at all submaximal

239 work rates between modalities (Table 1, Fig. 1).

240 Although participants reached their $\mathrm{T}_{\text {vent }}$ at the same absolute work rate during treadmill

241 vs. cycle testing ( $91 \pm 6$ vs. $92 \pm 6$ watts, $\mathrm{p}>0.05$ ), mean values of $\dot{\mathrm{V}} \mathrm{O}_{2}, \dot{\mathrm{V} C O}{ }_{2}, \mathrm{HR}$ and the $\mathrm{O}_{2}$

242 pulse were higher, while RER was lower at $\mathrm{T}_{\text {vent }}$ during treadmill exercise (Table 1).

$243 \quad$ Ventilatory, breathing pattern and operating lung volume responses. Despite significant

244 differences in $\dot{\mathrm{VO}}_{2}$ and $\dot{\mathrm{V}} \mathrm{CO}_{2}$ at the peak of treadmill vs. cycle exercise, $\dot{\mathrm{V}}_{\mathrm{E}}, \mathrm{V}_{\mathrm{T}}, f_{\mathrm{R}}$, IC and IRV

245 were not different between modalities at end-exercise (Table 1, Fig. 2).

246 As illustrated in Fig. 2, mean values of $\dot{V}_{\mathrm{E}}$ were higher at all standardized submaximal

247 work rates $\geq 75$ watts (including $T_{\text {vent }}$ ) during treadmill vs. cycle exercise testing; for example, by

$24817.4 \pm 5.6 \mathrm{Lmin}^{-1}$ or $21 \pm 7 \%$ at iso-work (Table 1, Fig. 2). The relatively greater $\dot{\mathrm{V}}_{\mathrm{E}}$ response to

249 treadmill vs. cycle exercise was associated with the adoption of a more rapid and shallow

250 breathing pattern (Fig. 2). Operating lung volumes were not different between modalities at any

251 standardized submaximal work rate and at peak exercise (Fig. 2). The relationship between

252 exercise-induced changes in IC and $\dot{\mathrm{V}}_{\mathrm{E}}$ was also similar between modalities (Fig. 2). In contrast,

253 there was a rightward shift of the IRV- $\dot{\mathrm{V}}_{\mathrm{E}}$ relationship during treadmill vs. cycle exercise (Fig.

254 2), such that mean values of $\dot{V}_{\mathrm{E}}$ were higher at any IRV during submaximal treadmill vs. cycle

255 exercise; for example, by $\sim 15-20 \mathrm{Lmin}^{-1}$ at IRV's ranging from 0.5 to $2.0 \mathrm{~L}$.

Diaphragm EMG and respiratory pressure responses. On average, EMGdi,max was not

257 different when evaluated on treadmill vs. cycle exercise test days: $238.2 \pm 19.8$ vs. $230.0 \pm 18.6$

$258 \mu \mathrm{V}$, respectively $(\mathrm{p}=0.740)$. The EMGdi,rms\%max was higher at the peak of treadmill vs. cycle

259 exercise (77.2 \pm 3.6 vs. $67.0 \pm 4.9)$; however, this difference did not reach statistical significance 
260 ( $\mathrm{p}=0.120)$ (Table 1, Fig. 3). Neither Pes,tidal, Pdi,tidal nor Pga,peak were different at the peak

261 of treadmill vs. cycle exercise (Table 1, Fig. 3).

262 As illustrated in Fig. 3, Pes,tidal was higher during treadmill vs. cycle testing at

263 standardized submaximal work rates $\geq 125$ watts (Fig. 3). Similarly, Pdi,tidal was higher at iso-

264 work, while Pga,peak was higher at 175 watts and at iso-work during treadmill vs. cycle testing

265 (Fig. 3). By contrast, EMGdi,rms\%max was not significantly different at any submaximal work

266 rate during treadmill vs. cycle exercise (Fig. 3).

267 Symptom responses. A higher percentage (60\% vs. 27\%) of our participants identified

268 intolerable leg discomfort as the main reason for stopping cycle vs. treadmill exercise. The

269 relative contribution of intolerable leg discomfort to exercise cessation was also higher at the end

270 of cycle vs. treadmill testing $(67.9 \pm 6.6 \%$ vs. $43.4 \pm 7.7 \%, \mathrm{p}=0.023)$. The majority of

271 participants self-selected descriptor phrases alluding to a heightened sense of 'work/effort of

272 breathing' and of 'rapid breathing' at the peak of both treadmill and cycle testing; for example,

273 'Breathing in requires effort' (cycle, 71\% vs. treadmill, 64\%), 'My breathing is heavy' (cycle,

$27479 \%$ vs. treadmill, 93\%), 'My breathing requires more work' (cycle, 71\% vs. treadmill, 93\%)

275 and 'I feel that my breathing is rapid' (cycle, 85\% vs. treadmill, 93\%).

276 Intensity ratings of leg discomfort were higher at iso-work and at peak exercise during

277 cycle vs. treadmill testing (by $0.9 \pm 0.5$ and $1.2 \pm 0.6$ Borg 0-10 scale units, respectively) (Table

278 1, Fig. 4). As illustrated in Fig. 4, the relationship between increasing intensity ratings of leg

279 discomfort and increasing $\dot{\mathrm{VO}}_{2}$ was displaced to the right during treadmill vs. cycle exercise.

280 Borg 0-10 scale intensity and unpleasantness ratings of dyspnea were not different

281 between modalities at any standardized submaximal work rate and at peak exercise (Table 1, Fig.

282 4). Compared to cycle testing, treadmill testing was associated with a rightward shift of the 
283 dyspnea intensity- $\dot{V}_{\mathrm{E}}$ relationship (Fig. 4). By contrast, the relationship between exercise-

284 induced changes in dyspnea intensity ratings and each of EMGdi,rms\%max and IRV were

285 relatively superimposed during treadmill and cycle ergometry (Fig. 4). A similar influence of

286 exercise mode was observed on the relationship exercise-induced changes in dyspnea

287 unpleasantness ratings and each of $\dot{\mathrm{V}}_{\mathrm{E}}$, EMGdi,rms\%max and IRV (data not shown).

\section{DISCUSSION}

290 The main findings of this study are that, despite significant between-test differences in $\dot{\mathrm{V}}_{2}$,

$291 \dot{\mathrm{V} C O}_{2}, \mathrm{RER}, \mathrm{HR}, \mathrm{O}_{2}$ pulse, $\dot{\mathrm{V}}_{\mathrm{E}}, f_{\mathrm{R}}$, and inspiratory and expiratory muscle pressures, detailed

292 assessments of ventilatory efficiency and gas exchange $\left(\dot{\mathrm{V}}_{\mathrm{E}} / \dot{\mathrm{VO}}_{2}, \dot{\mathrm{V}}_{\mathrm{E}} / \dot{\mathrm{V} C O} \mathrm{O}_{2}, \mathrm{P}_{\mathrm{ET}} \mathrm{O}_{2}, \mathrm{P}_{\mathrm{ET}} \mathrm{CO}_{2}\right)$,

293 dynamic operating lung volumes (IC, IRV), neural inspiratory drive (EMGdi,rms\%max), and

294 exertional symptoms (dyspnea, leg discomfort) were similar at all or most submaximal work

295 rates during incremental treadmill vs. cycle exercise testing.

296 The men in our study exerted maximal (or near maximal) effort during incremental cycle

297 and treadmill exercise testing as indicated by symptom-limited peak respiratory exchange ratio,

298 heart rate and symptom intensity values/ratings that were, on average, $\geq 1.06,>95 \%$ predicted

299 (cycle, $96.3 \pm 1.2 \%$; treadmill, $99.2 \pm 1.3 \%$ ) and $\sim 7-9$ Borg 0-10 scale units, respectively.

300 Consistent with previous reports (Duke et al. 2014; Faulkner et al. 1971; Hermansen et al. 1970;

301 Hermansen and Saltin 1969; Matsui et al. 1978; Miles et al. 1980; Miyamura and Honda 1972;

302 Porszasz et al. 2003; Tanner et al. 2014), $\mathrm{V}_{2}$ was higher at $\mathrm{T}_{\text {vent }}$ (by 23\%) and at the peak of

303 treadmill vs. cycle exercise testing (by $12 \%$ ), even though work rate was not different between

304 modalities at either measurement time. As previously discussed, these differences likely reflect

305 1) recruitment of a relatively larger skeletal muscle mass, with attainment of a higher maximal 
306 cardiac output, stroke volume, arteriovenous $\mathrm{O}_{2}$ difference, leg muscle blood flow and total

307 vascular conductance during treadmill running (Hermansen et al. 1970; Hermansen and Saltin

308 1969; Matsui et al. 1978; Miyamura and Honda 1972; Niederberger et al. 1974); 2) higher fat

309 and lower carbohydrate oxidation rates during submaximal and maximal treadmill running vs.

310 cycling (Achten et al. 2003; Cheneviere et al. 2010); 3) higher oxidative relative to glycolytic

311 enzyme activity in skeletal muscles predominantly used in running vs. those predominantly used

312 in cycling (Jacobs and Sjodin 1985); and 4) less rapid rate of development of metabolic acidosis

313 during treadmill vs. cycle ergometry (Koyal et al. 1976; Okita et al. 1998). Indeed, RER was

314 lower, while $\mathrm{V} \mathrm{CO}_{2}$, $\mathrm{HR}$ and the $\mathrm{O}_{2}$ pulse (a surrogate measure of stroke volume (Bhambhani et

315 al. 1994)) were higher during treadmill vs. cycle ergometry at $T_{\text {vent }}$, iso-work and at peak

316 exercise.

317 It is clear from Fig. 1 that cardiometabolic responses were higher during incremental

318 treadmill vs. cycle exercise at most standardized submaximal work rates. These findings, which

319 substantiate the results of Porszasz et al. (2003) in health and Ciavaglia et al. (2014a; 2014b) in

320 COPD, suggest that treadmill walking/running is less metabolically efficient than stationary

321 cycling. Muscles of the upper body (e.g., arms, shoulders) and trunk (e.g., rectus abdominis) are

322 presumably more active during treadmill vs. cycle exercise. Although activation of these muscle

323 groups cost metabolic energy, they do not contribute to the production of external mechanical

324 power during treadmill walking/running. It follows that, in our study, the widening disparity

325 between work rate and each of $\dot{\mathrm{V}}_{2}, \mathrm{~V} \mathrm{CO}_{2}, \mathrm{HR}$ and the $\mathrm{O}_{2}$ pulse during treadmill vs. cycle

326 exercise may reflect, at least in part, relatively greater metabolic activity of the upper body

327 and/or trunk muscles as work rate increased during treadmill exercise. 
329 study, neither $\dot{\mathrm{V}}_{\mathrm{E}}$ nor $\dot{\mathrm{V}} \mathrm{CO}_{2}$ was significantly different between modalities at standardized work

330 rates below $\mathrm{T}_{\text {vent. }}$ By contrast, $\dot{\mathrm{V}}_{\mathrm{E}}$ and $\dot{\mathrm{V}} \mathrm{CO}_{2}$ were significantly and proportionately higher

331 during treadmill vs. cycle exercise at $\mathrm{T}_{\text {vent }}$ and at submaximal work rates above $\mathrm{T}_{\text {vent. }}$ Under

332 these circumstances, neither $\dot{\mathrm{V}}_{\mathrm{E}} / \dot{\mathrm{VCO}}_{2}$ nor $\mathrm{P}_{\mathrm{ET}} \mathrm{CO}_{2}$ was significantly different at any submaximal

333 work rate across modalities. Thus, ventilatory efficiency was similar during treadmill vs. cycle

334 exercise testing when the protocols were matched for increments in work rate.

335 In keeping with the results of Kalsas and Thorsen (2009) and Elliott and Grace (2010),

336 the greater $\dot{\mathrm{V}}_{\mathrm{E}}$ observed during treadmill vs. cycle exercise at $\mathrm{T}_{\mathrm{vent}}$ and at submaximal work rates

337 above $\mathrm{T}_{\mathrm{vent}}$ was the result of a greater $f_{\mathrm{R}}$ with no difference in $\mathrm{V}_{\mathrm{T}}$. As such, $f_{\mathrm{R}}$ was higher, while

$338 \mathrm{~V}_{\mathrm{T}}$ was lower at any $\dot{\mathrm{V}}_{\mathrm{E}}$ throughout much of treadmill vs. cycle exercise. Despite significant

339 differences in the $\dot{\mathrm{V}}_{\mathrm{E}}$-work rate relationship during treadmill vs. cycle ergometry, exercise mode

340 had no effect on the relationship between IC and each of work rate and $\dot{V}_{\mathrm{E}}$. Under these

341 circumstances, adoption of a more rapid and shallow breathing pattern during treadmill vs. cycle

342 exercise likely helped to preserve the IRV-work rate relationship between modalities.

343 Our study is the first to examine the effect of exercise mode on neural inspiratory drive

344 and respiratory muscle pressure (effort) requirements in health. The higher demand for $\dot{V}_{\mathrm{E}}$

345 during treadmill vs. cycle exercise at submaximal work rates above $\mathrm{T}_{\text {vent }}$ was supported by

346 greater Pes,tidal, Pdi,tidal and Pga,peak. Despite differences in $\dot{\mathrm{V}}_{\mathrm{E}}$ and respiratory muscle

347 pressures between modalities, EMGdi,rms\%max was not different at any submaximal work rate

348 during treadmill vs. cycle exercise. By adopting a more rapid and shallow breathing pattern, our

349 participants maintained a larger IRV (by $\sim 200-300 \mathrm{~mL}$ ) for any given $\dot{\mathrm{V}}_{\mathrm{E}}$ throughout the majority

350 of treadmill vs. cycle exercise. We speculate that maintenance of a larger dynamic IRV was 
351 associated with less elastic loading and functional weakening of the inspiratory pump muscles

352 (e.g., diaphragm) for any given $\dot{\mathrm{V}}_{\mathrm{E}}$ during treadmill testing. We further speculate that, for these

353 reasons, exercise mode had no effect on EMGdi,rms\%max-work rate relationships.

$354 \quad$ Mean values of $\dot{\mathrm{V}}_{\mathrm{E}}, \mathrm{V}_{\mathrm{T}}, f_{\mathrm{R}}, \mathrm{IC}, \mathrm{IRV}$, Pes,tidal, Pdi,tidal and Pga,peak were not different at

355 the peak of incremental treadmill vs. cycle testing, even though $\dot{\mathrm{V}} \mathrm{O}_{2}, \dot{\mathrm{VCO}}{ }_{2}$ and

356 EMGdi,rms\%max were 8-15\% higher in the former. These findings corroborate those of Clark

357 et al. (1980), Johnson et al. (1992) and McClaran et al. (1999) who found that increased

358 chemostimulation via hypercapnia did not elicit further increases in $\dot{\mathrm{V}}_{\mathrm{E}}$ and/or peak inspiratory

359 and expiratory Pes during treadmill and cycle exercise testing near the limits of tolerance in

360 young, endurance trained men with maximal $\dot{\mathrm{VO}}_{2}$ values of $63-73 \mathrm{~mL} \mathrm{~kg}^{-1} \mathrm{~min}^{-1}$. Collectively,

361 these findings support the existence of critical dynamic mechanical constraints on $\dot{\mathrm{V}}_{\mathrm{E}}$ during

362 treadmill and cycle exercise near the limits of tolerance in healthy, young, fit men with normal

363 lung function.

364 A study of 20 healthy young adults by Sharma et al. (2015) recently reported that 1)

365 intensity ratings of leg fatigue and dyspnea were not different during treadmill vs. cycle exercise

366 at any standardized submaximal $\dot{\mathrm{VO}}_{2}$ ranging from 1.0 to $2.5 \mathrm{Lmin}^{-1}$; and 2) exercise mode had

367 no effect dyspnea intensity- $\dot{V}_{\mathrm{E}}$ relationships. In our study, intensity ratings of leg discomfort

368 were similar between modalities up to 175 watts, but became greater at iso-work and at the peak

369 of cycle vs. treadmill exercise. Moreover, the relative contribution of intolerable leg discomfort

370 to exercise cessation was higher at the peak of cycle vs. treadmill testing. It follows that a higher

371 percentage of our participants stopped cycle vs. treadmill exercise because of intolerable leg

372 discomfort. We speculate that these differences reflect the sensory consequences associated with

373 relatively greater neural activation of the lower limbs needed to produce equivalent external 
374 work rates near the limits of tolerance during cycle vs. treadmill testing. Indeed, Bijker et al.

375 (2002) found that the magnitude of the increase in vastus lateralis EMG and biceps femoris EMG

376 needed to support any given increase in work rate was much greater during cycling compared to

377 running in a group of 11 healthy young adults.

378 In contrast to Sharma et al. (2015), we observed a rightward shift of the leg discomfort-

$379 \dot{\mathrm{VO}}_{2}$ relationship during treadmill vs. cycle ergometry; that is, intensity ratings of leg discomfort

380 were much lower at any standardized $\mathrm{V}_{2}$ during treadmill vs. cycle testing (e.g., by $\sim 3$ Borg 0-

38110 scale units at a $\dot{V}_{2}$ of $50 \mathrm{~mL} \mathrm{~kg}^{-1} \mathrm{~min}^{-1}$ ). These differences may reflect the development of a

382 more severe metabolic acidosis for any given $\dot{\mathrm{V}}_{2}$ during cycle ergometry (Koyal et al. 1976;

383 Okita et al. 1998) and/or progressively greater activation of the upper body and trunk muscles

384 that do not contribute to the production of external mechanical power (and, by extension, the 385 perception of leg discomfort), but nevertheless cost metabolic energy during treadmill running.

386 Despite the aforementioned differences in $\dot{\mathrm{V}}_{\mathrm{E}}$ and respiratory muscle pressure responses

387 between the two modalities, Borg 0-10 scale intensity (and unpleasantness) ratings of dyspnea

388 were identical at submaximal work rates and at peak exercise during treadmill vs. cycle testing.

389 Consequently, and in contrast to the results of Sharma et al. (2015), dyspnea intensity (and 390 unpleasantness)- $\dot{\mathrm{V}}_{\mathrm{E}}$ relationships were shifted to the right throughout the majority treadmill vs.

391 cycle exercise testing. Accumulating evidence suggests that neural inspiratory drive (and the 392 attendant 'central corollary discharge') is the proximate source of exertional dyspnea in health 393 (Jensen et al. 2011; Mendonca et al. 2014; Schaeffer et al. 2014) and in patients with COPD

394 (Ciavaglia et al. 2014a; Guenette et al. 2014; Jolley et al. 2015). It follows that preservation of 395 the EMGdi,rms\%max-work rate relationship during treadmill vs. cycle testing in our study was

396 likely responsible for preservation of the dyspnea intensity (and unpleasantness)-work rate 
397 relationship, despite significant differences in $\dot{\mathrm{V}}_{\mathrm{E}}$ and contractile respiratory muscle pressure

398 (effort) requirements. Indeed, exercise mode had no effect on dyspnea intensity (and

399 unpleasantness)-EMGdi,rms\%max relationships.

400 It is possible that the observed rightward shift of the dyspnea intensity (and

401 unpleasantness)- $\dot{\mathrm{V}}_{\mathrm{E}}$ relationship during treadmill vs. cycle exercise may also reflect, at least in

402 part, temporal desensitization to the sensory consequences of increased $\dot{\mathrm{V}}_{\mathrm{E}}$ and contractile

403 respiratory muscle effort requirements during a form of exercise (walking/running) that was

404 more familiar to and/or preferred by our participants. This hypothesis is bolstered by the results

405 of Schneider et al. (2009) and Brummer et al. (2011) who found that familiar and/or preferred

406 exercise modes (e.g., treadmill running) are associated with decreased neuronal activity

407 (deactivation) in the frontal cortex of healthy recreational runners, where increased neural

408 activation of the prefrontal cortex has been mechanistically linked to the perception of activity-

409 related dyspnea in health and COPD (Higashimoto et al. 2011).

410

411 Methodological considerations. Our treadmill protocol employed linear increases in grade and

412 curvilinear increases in speed, which is in contrast to previous studies that utilized linear

413 increases in speed and curvilinear increases in grade (Ciavaglia et al. 2014a; Ciavaglia et al.

414 2014b; Porszasz et al. 2003). Despite these differences, all studies reported 1) a linear $\dot{\mathrm{V}} \mathrm{O}_{2}$-work

415 rate relationship during treadmill testing and 2) that the $\mathrm{VO}_{2}$-work rate slope was higher during 416 treadmill vs. cycle exercise.

417 Our participants were healthy, young, non-obese men with normal-to-above normal

418 levels of cardiorespiratory fitness. As such, our results may not be generalizable to elderly men 
419 and women; obese men and women; physically inactive/deconditioned men and women; patients

420 with chronic cardiorespiratory and/or neuromuscular disease; or any combination thereof.

421 Training specificity may influence comparisons of the physiological response to treadmill

422 vs. cycle exercise. For example, Verstappen et al. (1982) found that maximal $\dot{\mathrm{VO}}_{2}$ values were

$42314 \%$ higher during treadmill vs. cycle ergometry in runners, but not significantly different

424 between modalities in cyclists. Although detailed assessments of training history were not

425 recorded in our study, all of our participants were recreationally active and none were known to

426 be training for participation in any competitive athletic event(s) and/or involved in bicycling as a

427 sport or common form of recreation. Nevertheless, we cannot rule out the possibility that

428 unmeasured differences in our participants' training histories may have influenced our results.

429 In our study, stride frequency during treadmill testing and pedal cadence during cycle

430 testing were not matched within- or between-subjects. Thus, maintenance of a relatively higher

431 stride frequency vs. pedal cadence may have contributed to differences in the $f_{\mathrm{R}}$-work rate

432 relationship during treadmill vs. cycle exercise (Caretti et al. 1992).

434 Summary and implications. In summary: 1) cardiometabolic, ventilation and respiratory 435 muscle pressure (effort) responses were consistently higher at standardized submaximal work 436 rates during incremental treadmill vs. cycle exercise; and 2) with few exceptions, detailed 437 assessments of ventilatory efficiency, dynamic operating lung volumes, neural inspiratory drive, 438 dyspnea and leg discomfort were similar at equivalent submaximal work rates during treadmill 439 vs. cycle ergometry.

440 Our findings suggest that physiological parameters relevant to the prescription of exercise 441 - specifically peak $\dot{\mathrm{VO}}_{2}$, peak $\mathrm{HR}$ and $\dot{\mathrm{VO}}_{2}$ at $\mathrm{T}_{\text {vent }}$ - should be assessed in each exercise mode for 
442 optimal (mode-specific) training intensity determination. Alternatively, the lack of effect of

443 exercise mode on peak work rate in our study advocates for the use of this readily available

444 parameter to determine optimal training intensity, regardless of exercise training mode.

445 In terms of evaluating an individuals' occupational readiness, our finding of a higher

446 peak $\dot{\mathrm{V}}_{2}$ on treadmill vs. cycle exercise testing may lead to the identification of occupational

447 tasks with higher metabolic equivalents and that may be unsafe for some adults. By contrast, our

448 finding of a lower peak $\mathrm{V}_{2}$ on cycle vs. treadmill exercise testing may lead to the identification

449 of occupational tasks with metabolic equivalents deemed as "safe" but below the physical

450 requirements of a particular position of employment.

451 Finally, peak $\dot{\mathrm{VO}}_{2}$ is often used to evaluate pre-operative risk for major surgery, with

452 values $<14 \mathrm{mLkg}^{-1} \mathrm{~min}^{-1}$ being indicative of "high-risk" (American Thoracic and American

453 College of Chest 2003). In our study, peak $\mathrm{VO}_{2}$ was, on average, $6.5 \mathrm{mLkg}^{-1} \mathrm{~min}^{-1}$ (or 12\%)

454 higher when testing was performed on the treadmill vs. cycle ergometer. It follows that, in a

455 given surgical candidate, peak $\dot{\mathrm{VO}}_{2}$ measured pre-operatively on a cycle ergometer may indicate

456 "high-risk", while peak $\mathrm{V}_{2}$ measured pre-operatively on a treadmill may indicate "low-to-

457 moderate risk". 
463 Acknowledgements. The authors would like to thank the participants for their time and

464 cooperation. Financial support was provided to D.J. by the Natural Sciences and Engineering

465 Research Council of Canada (RGPIN 402598-2011). C.T.M. was supported by a Canadian

466 Institutes of Health Research-Québec Respiratory Health Training Program Award. D.J. was

467 supported by a Chercheurs-Boursiers Junior 1 salary award from the Fonds de Recherche du

468 Québec-Santé and by a William Dawson Research Scholars Award (McGill University).

470 Author contributions. Conception and design: D.J.; Data collection and analysis: K.M.M., 471 H.G.K., C.A.W-M., M.R.S., C.T.M.; Data analysis: K.M.M., H.G.K., C.A.W-M., M.R.S.,

472 C.T.M., D.J.; Interpretation: K.M.M., D.J.; Drafting the manuscript for important intellectual 473 content: K.M.M., D.J.

475 Disclosures. None of the authors has a real or perceived conflict of interest to disclose. The 476 sponsor had no role in the conduct of the study, data analysis, manuscript preparation, or 477 manuscript review. 


\section{REFERENCES}

480 Achten, J., Venables, M.C., and Jeukendrup, A.E. 2003. Fat oxidation rates are higher during

481 running compared with cycling over a wide range of intensities. Metabolism: clinical and 482 experimental 52(6): 747-752.

483 American Thoracic, S., and American College of Chest, P. 2003. ATS/ACCP Statement on 484 cardiopulmonary exercise testing. American journal of respiratory and critical care 485 medicine 167(2): 211-277. doi: 10.1164/rccm.167.2.211.

486 Beaver, W.L., Wasserman, K., and Whipp, B.J. 1986. A new method for detecting anaerobic 487 threshold by gas exchange. J Appl Physiol (1985) 60(6): 2020-2027.

488 Bhambhani, Y., Norris, S., and Bell, G. 1994. Prediction of stroke volume from oxygen pulse 489 measurements in untrained and trained men. Canadian journal of applied physiology $=$ 490 Revue canadienne de physiologie appliquee 19(1): 49-59.

491 Bijker, K.E., de Groot, G., and Hollander, A.P. 2002. Differences in leg muscle activity during 492 running and cycling in humans. European journal of applied physiology 87(6): 556-561. 493 doi: $10.1007 / \mathrm{s} 00421-002-0663-8$.

494 Borg, G.A. 1982. Psychophysical bases of perceived exertion. Medicine and science in sports 495 and exercise 14(5): 377-381.

496 Brummer, V., Schneider, S., Abel, T., Vogt, T., and Struder, H.K. 2011. Brain cortical activity is 497 influenced by exercise mode and intensity. Medicine and science in sports and exercise 498 43(10): 1863-1872. doi: 10.1249/MSS.0b013e3182172a6f.

499 Caretti, D.M., Szlyk, P.C., and Sils, I.V. 1992. Effects of exercise modality on patterns of 500 ventilation and respiratory timing. Respiration physiology 90(2): 201-211. 
501 Cheneviere, X., Malatesta, D., Gojanovic, B., and Borrani, F. 2010. Differences in whole-body

502 fat oxidation kinetics between cycling and running. European journal of applied physiology

503 109(6): 1037-1045. doi: 10.1007/s00421-010-1443-5.

504 Ciavaglia, C.E., Guenette, J.A., Langer, D., Webb, K.A., Alberto Neder, J., and O'Donnell, D.E.

505 2014a. Differences in respiratory muscle activity during cycling and walking do not

506 influence dyspnea perception in obese patients with COPD. J Appl Physiol (1985) 117(11):

507 1292-1301. doi: 10.1152/japplphysiol.00502.2014.

508 Ciavaglia, C.E., Guenette, J.A., Ora, J., Webb, K.A., Neder, J.A., and O'Donnell, D.E. 2014b. Does

509 exercise test modality influence dyspnoea perception in obese patients with COPD? The

510 European respiratory journal : official journal of the European Society for Clinical

511 Respiratory Physiology 43(6): 1621-1630. doi: 10.1183/09031936.00151513.

512 Clark, J.M., Sinclair, R.D., and Lenox, J.B. 1980. Chemical and nonchemical components of

513 ventilation during hypercapnic exercise in man. Journal of applied physiology: respiratory,

514 environmental and exercise physiology 48(6): 1065-1076.

515 Cumming, G.R. 1977. Comparison of treadmill and bicycle ergometer exercise in middle516 aged males. American heart journal 93(2): 261.

517 Duke, J.W., Stickford, J.L., Weavil, J.C., Chapman, R.F., Stager, J.M., and Mickleborough, T.D.

518 2014. Operating lung volumes are affected by exercise mode but not trunk and hip angle

519 during maximal exercise. European journal of applied physiology 114(11): 2387-2397. doi:

$520 \quad 10.1007 / \mathrm{s} 00421-014-2956-0$.

521 Elliott, A.D., and Grace, F. 2010. An examination of exercise mode on ventilatory patterns

522 during incremental exercise. European journal of applied physiology 110(3): 557-562. doi: $523 \quad 10.1007 / \mathrm{s} 00421-010-1541-4$. 
524 Faulkner, J.A., Roberts, D.E., Elk, R.L., and Conway, J. 1971. Cardiovascular responses to

525 submaximum and maximum effort cycling and running. J Appl Physiol 30(4): 457-461.

526 Guenette, J.A., Chin, R.C., Cheng, S., Dominelli, P.B., Raghavan, N., Webb, K.A., Neder, J.A., and

527 O'Donnell, D.E. 2014. Mechanisms of exercise intolerance in global initiative for chronic

528 obstructive lung disease grade 1 COPD. The European respiratory journal : official journal

529 of the European Society for Clinical Respiratory Physiology 44(5): 1177-1187. doi:

$530 \quad 10.1183 / 09031936.00034714$.

531 Hankinson, J.L., Odencrantz, J.R., and Fedan, K.B. 1999. Spirometric reference values from a

532 sample of the general U.S. population. American journal of respiratory and critical care

533 medicine 159(1): 179-187.

534 Haouzi, P. 2006. Theories on the nature of the coupling between ventilation and gas

535 exchange during exercise. Respiratory physiology \& neurobiology 151(2-3): 267-279. doi:

536 10.1016/j.resp.2005.11.013.

537 Hermansen, L., Ekblom, B., and Saltin, B. 1970. Cardiac output during submaximal and

538 maximal treadmill and bicycle exercise. J Appl Physiol 29(1): 82-86.

539 Hermansen, L., and Saltin, B. 1969. Oxygen uptake during maximal treadmill and bicycle

540 exercise. J Appl Physiol 26(1): 31-37.

541 Higashimoto, Y., Honda, N., Yamagata, T., Matsuoka, T., Maeda, K., Satoh, R., Nishiyama, O.,

542 Sano, H., Iwanaga, T., Miyara, T., Muraki, M., Tomita, K., Kume, H., Miyai, I., Tohda, Y., and

543 Fukuda, K. 2011. Activation of the prefrontal cortex is associated with exertional dyspnea

544 in chronic obstructive pulmonary disease. Respiration; international review of thoracic

545 diseases 82(6): 492-500. doi: 10.1159/000324571. 
546 Jacobs, I., and Sjodin, B. 1985. Relationship of ergometer-specific VO2 max and muscle

547 enzymes to blood lactate during submaximal exercise. Br J Sports Med 19(2): 77-80.

548 Jensen, D., O'Donnell, D.E., Li, R., and Luo, Y.M. 2011. Effects of dead space loading on neuro-

549 muscular and neuro-ventilatory coupling of the respiratory system during exercise in

550 healthy adults: implications for dyspnea and exercise tolerance. Respiratory physiology \&

551 neurobiology 179(2-3): 219-226. doi: 10.1016/j.resp.2011.08.009.

552 Johnson, B.D., Saupe, K.W., and Dempsey, J.A. 1992. Mechanical constraints on exercise

553 hyperpnea in endurance athletes. J Appl Physiol (1985) 73(3): 874-886.

554 Jolley, C.J., Luo, Y.M., Steier, J., Rafferty, G.F., Polkey, M.I., and Moxham, J. 2015. Neural

555 respiratory drive and breathlessness in COPD. The European respiratory journal : official

556 journal of the European Society for Clinical Respiratory Physiology 45(2): 355-364. doi:

$557 \quad 10.1183 / 09031936.00063014$.

558 Jones, N. 1997. Clinical Exercise Testing. W.B Saunders Company, Philadelphia.

559 Kalsas, K., and Thorsen, E. 2009. Breathing patterns during progressive incremental cycle

560 and treadmill exercise are different. Clinical physiology and functional imaging 29(5): 335-

561 338. doi: 10.1111/j.1475-097X.2009.00874.x.

562 Kim, S., Yamabe, H., and Yokoyama, M. 1999. Hemodynamic characteristics during treadmill

563 and bicycle exercise in chronic heart failure: mechanism for different responses of peak

564 oxygen uptake. Jpn Circ J 63(12): 965-970.

565 Koyal, S.N., Whipp, B.J., Huntsman, D., Bray, G.A., and Wasserman, K. 1976. Ventilatory

566 responses to the metabolic acidosis of treadmill and cycle ergometry. J Appl Physiol 40(6):

$567 \quad 864-867$. 
568 Matsui, H., Kitamura, K., and Miyamura, M. 1978. Oxygen uptake and blood flow of the

569 lower limb in maximal treadmill and bicycle exercise. European journal of applied

570 physiology and occupational physiology 40(1): 57-62.

571 McClaran, S.R., Wetter, T.J., Pegelow, D.F., and Dempsey, J.A. 1999. Role of expiratory flow

572 limitation in determining lung volumes and ventilation during exercise. Journal of applied

573 physiology 86(4): 1357-1366.

574 Mendonca, C.T., Schaeffer, M.R., Riley, P., and Jensen, D. 2014. Physiological mechanisms of

575 dyspnea during exercise with external thoracic restriction: role of increased neural

576 respiratory drive. J Appl Physiol (1985) 116(5): 570-581. doi:

577 10.1152/japplphysiol.00950.2013.

578 Miles, D.S., Critz, J.B., and Knowlton, R.G. 1980. Cardiovascular, metabolic, and ventilatory

579 responses of women to equivalent cycle ergometer and treadmill exercise. Medicine and

580 science in sports and exercise 12(1): 14-19.

581 Miller, M.R., Hankinson, J., Brusasco, V., Burgos, F., Casaburi, R., Coates, A., Crapo, R., Enright,

582 P., van der Grinten, C.P., Gustafsson, P., Jensen, R., Johnson, D.C., MacIntyre, N., McKay, R.,

583 Navajas, D., Pedersen, O.F., Pellegrino, R., Viegi, G., and Wanger, J. 2005. Standardisation of

584 spirometry. The European respiratory journal : official journal of the European Society for

585 Clinical Respiratory Physiology 26(2): 319-338. doi: 10.1183/09031936.05.00034805.

586 Miyamura, M., and Honda, Y. 1972. Oxygen intake and cardiac output during maximal 587 treadmill and bicycle exercise. J Appl Physiol 32(2): 185-188.

588 Niederberger, M., Bruce, R.A., Kusumi, F., and Whitkanack, S. 1974. Disparities in 589 ventilatory and circulatory responses to bicycle and treadmill exercise. Br Heart J 36(4): $590 \quad 377-382$. 
591 O'Donnell, D.E., Hong, H.H., and Webb, K.A. 2000. Respiratory sensation during chest wall

592 restriction and dead space loading in exercising men. J Appl Physiol 88(5): 1859-1869.

593 Okita, K., Nishijima, H., Yonezawa, K., Ohtsubo, M., Hanada, A., Kohya, T., Murakami, T., and

594 Kitabatake, A. 1998. Skeletal muscle metabolism in maximal bicycle and treadmill exercise

595 distinguished by using in vivo metabolic freeze method and phosphorus-31 magnetic

596 resonance spectroscopy in normal men. Am J Cardiol 81(1): 106-109.

597 Pannier, J.L., Vrijens, J., and Van Cauter, C. 1980. Cardiorespiratory response to treadmill

598 and bicycle exercise in runners. European journal of applied physiology and occupational

599 physiology 43(3): 243-251.

600 Parshall, M.B., Schwartzstein, R.M., Adams, L., Banzett, R.B., Manning, H.L., Bourbeau, J.,

601 Calverley, P.M., Gift, A.G., Harver, A., Lareau, S.C., Mahler, D.A., Meek, P.M., and O'Donnell,

602 D.E. 2012. An official American Thoracic Society statement: update on the mechanisms, 603 assessment, and management of dyspnea. American journal of respiratory and critical care 604 medicine 185(4): 435-452. doi: 10.1164/rccm.201111-2042ST.

605 Porszasz, J., Casaburi, R., Somfay, A., Woodhouse, L.J., and Whipp, B.J. 2003. A treadmill 606 ramp protocol using simultaneous changes in speed and grade. Medicine and science in 607 sports and exercise 35(9): 1596-1603. doi: 10.1249/01.MSS.0000084593.56786.DA.

608 Schaeffer, M.R., Mendonca, C.T., Levangie, M.C., Andersen, R.E., Taivassalo, T., and Jensen, D. 609 2014. Physiological mechanisms of sex differences in exertional dyspnoea: role of neural 610 respiratory motor drive. Experimental physiology 99(2): 427-441. doi: 611 10.1113/expphysiol.2013.074880. 
612 Schneider, S., Brummer, V., Abel, T., Askew, C.D., and Struder, H.K. 2009. Changes in brain

613 cortical activity measured by EEG are related to individual exercise preferences. Physiol

614 Behav 98(4): 447-452. doi: 10.1016/j.physbeh.2009.07.010.

615 Sharma, P., Morris, N.R., and Adams, L. 2015. The effect of different exercise modalities on

616 dyspnea and leg fatigue in healthy subjects. Respiratory physiology \& neurobiology 210:

617 44-50. doi: 10.1016/j.resp.2015.02.001.

618 Stubbing, D.G., Pengelly, L.D., Morse, J.L., and Jones, N.L. 1980. Pulmonary mechanics during 619 exercise in normal males. J Appl Physiol 49(3): 506-510.

620 Tanner, D.A., Duke, J.W., and Stager, J.M. 2014. Ventilatory patterns differ between maximal 621 running and cycling. Respiratory physiology \& neurobiology 191: 9-16. doi: 622 10.1016/j.resp.2013.10.011.

623 Verstappen, F.T., Huppertz, R.M., and Snoeckx, L.H. 1982. Effect of training specificity on 624 maximal treadmill and bicycle ergometer exercise. International journal of sports medicine 625 3(1): 43-46. doi: 10.1055/s-2008-1026061.

626 Vestbo, J., Hurd, S.S., Agusti, A.G., Jones, P.W., Vogelmeier, C., Anzueto, A., Barnes, P.J., Fabbri, 627 L.M., Martinez, F.J., Nishimura, M., Stockley, R.A., Sin, D.D., and Rodriguez-Roisin, R. 2013. 628 Global strategy for the diagnosis, management, and prevention of chronic obstructive 629 pulmonary disease: GOLD executive summary. American journal of respiratory and critical 630 care medicine 187(4): 347-365. doi: 10.1164/rccm.201204-0596PP. 631 
Table 1. Effect of modality on physiological and perceptual responses at rest and during symptom-limited incremental exercise testing in 15 healthy, young men.

\begin{tabular}{|c|c|c|c|c|c|c|c|c|}
\hline \multirow[b]{2}{*}{ Parameter } & \multicolumn{2}{|c|}{ Rest } & \multicolumn{2}{|c|}{ Ventilatory Threshold } & \multicolumn{2}{|c|}{ Iso-work } & \multicolumn{2}{|c|}{ Peak } \\
\hline & Cycle & Treadmill & Cycle & Treadmill & Cycle & Treadmill & Cycle & Treadmill \\
\hline \multicolumn{9}{|l|}{ Cardiometabolic and gas exchange parameters } \\
\hline$\dot{\mathrm{VO}} \mathrm{O}_{2}\left(\mathrm{Lmin}^{-1}\right)$ & $0.40 \pm 0.03$ & $0.46 \pm 0.03$ & $1.68 \pm 0.06$ & $2.06 \pm 0.08 *$ & $3.87 \pm 0.15$ & $4.53 \pm 0.18^{*}$ & $4.24 \pm 0.14$ & $4.74 \pm 0.16^{*}$ \\
\hline$\dot{\mathrm{V}} \mathrm{O}_{2}\left(\mathrm{mLkg}^{-1} \cdot \mathrm{min}^{-1}\right)$ & $5.1 \pm 0.3$ & $5.9 \pm 0.3$ & $22.0 \pm 1.0$ & $26.9 \pm 1.0^{*}$ & $50.1 \pm 1.4$ & $58.8 \pm 1.8^{*}$ & $55.0 \pm 1.3$ & $61.5 \pm 1.5^{*}$ \\
\hline$\dot{\mathrm{V} C O}{ }_{2}\left(\mathrm{~mL} \mathrm{~kg}^{-1} \mathrm{~min}^{-1}\right)$ & $4.3 \pm 0.3$ & $4.5 \pm 0.3$ & $18.5 \pm 1.1$ & $21.2 \pm 1.1^{*}$ & $52.1 \pm 1.8$ & $60.1 \pm 1.9^{*}$ & $60.4 \pm 1.9$ & $64.9 \pm 1.8^{*}$ \\
\hline RER & $0.84 \pm 0.03$ & $0.76 \pm 0.02 *$ & $0.83 \pm 0.02$ & $0.78 \pm 0.01 *$ & $1.04 \pm 0.01$ & $1.02 \pm 0.02$ & $1.10 \pm 0.02$ & $1.06 \pm 0.02 *$ \\
\hline Heart rate (beats $\min ^{-1}$ ) & $80.9 \pm 2.9$ & $87.8 \pm 4.4^{*}$ & $116.1 \pm 2.8$ & $122.9 \pm 3.8^{*}$ & $178.9 \pm 2.7$ & $190.1 \pm 2.1 *$ & $188.1 \pm 2.3$ & $193.8 \pm 2.5^{*}$ \\
\hline $\mathrm{O}_{2}$ pulse $\left(\mathrm{ml} \mathrm{O}_{2}\right.$ beat $\left.^{-1}\right)$ & $5.0 \pm 0.3$ & $5.4 \pm 0.5$ & $14.6 \pm 0.6$ & $16.9 \pm 0.7^{*}$ & $21.7 \pm 0.8$ & $23.9 \pm 1.1^{*}$ & $22.6 \pm 0.7$ & $24.5 \pm 1.0^{*}$ \\
\hline$\dot{\mathrm{V}}_{\mathrm{E}} / \dot{\mathrm{V}}_{2}$ & $32.8 \pm 1.3$ & $29.5 \pm 1.3 *$ & $21.0 \pm 0.5$ & $20.0 \pm 0.4$ & $27.4 \pm 1.0$ & $27.5 \pm 0.4$ & $33.5 \pm 1.1$ & $29.9 \pm 0.6^{*}$ \\
\hline$\dot{\mathrm{V}}_{\mathrm{E}} / \dot{\mathrm{VCO}}_{2}$ & $39.3 \pm 1.3$ & $38.9 \pm 1.4$ & $25.2 \pm 0.5$ & $25.6 \pm 0.5$ & $26.3 \pm 0.8$ & $27.0 \pm 0.4$ & $30.6 \pm 0.9$ & $28.4 \pm 0.4$ \\
\hline $\mathrm{P}_{\mathrm{ET}} \mathrm{O}_{2}(\mathrm{mmHg})$ & $107.0 \pm 1.8$ & $103.8 \pm 1.4^{*}$ & $98.6 \pm 1.1$ & $95.1 \pm 1.1$ & $107.3 \pm 1.2$ & $107.7 \pm 0.6$ & $114.0 \pm 0.9$ & $110.4 \pm 0.7 *$ \\
\hline $\mathrm{P}_{\mathrm{ET}} \mathrm{CO}_{2}(\mathrm{mmHg})$ & $34.8 \pm 1.0$ & $34.6 \pm 0.8$ & $43.1 \pm 0.7$ & $42.4 \pm 0.8$ & $40.8 \pm 1.1$ & $39.8 \pm 0.7^{*}$ & $35.5 \pm 0.9$ & $37.9 \pm 0.4^{*}$ \\
\hline \multicolumn{9}{|c|}{ Ventilation, breathing pattern and operating lung volume parameters } \\
\hline$\dot{\mathrm{V}}_{\mathrm{E}}\left({\left.\mathrm{L} \cdot \mathrm{min}^{-1}\right)}^{-1}\right.$ & $12.9 \pm 0.9$ & $13.4 \pm 0.9$ & $35.2 \pm 1.5$ & $41.2 \pm 1.9^{*}$ & $107.0 \pm 6.9$ & $124.4 \pm 4.8^{*}$ & $142.1 \pm 6.2$ & $141.0 \pm 3.9$ \\
\hline $\mathrm{V}_{\mathrm{T}}(\mathrm{L})$ & $0.99 \pm 0.07$ & $0.86 \pm 0.06$ & $1.82 \pm 0.11$ & $1.82 \pm 0.12$ & $3.04 \pm 0.14$ & $2.95 \pm 0.10$ & $3.08 \pm 0.08$ & $2.96 \pm 0.10$ \\
\hline$f_{\mathrm{R}}$ (breaths $\min ^{-1}$ ) & $14.0 \pm 0.8$ & $17.2 \pm 1.3 *$ & $20.7 \pm 1.3$ & $24.3 \pm 1.4^{*}$ & $35.7 \pm 1.9$ & $42.7 \pm 1.3^{*}$ & $46.4 \pm 1.6$ & $48.1 \pm 1.3$ \\
\hline $\mathrm{IC}(\mathrm{L})$ & $3.63 \pm 0.13$ & $3.71 \pm 0.14$ & $3.94 \pm 0.14$ & $3.99 \pm 0.13$ & $4.01 \pm 0.17$ & $3.96 \pm 0.13$ & $3.87 \pm 0.14$ & $3.70 \pm 0.15$ \\
\hline IRV (L) & $2.64 \pm 0.11$ & $2.84 \pm 0.14$ & $2.12 \pm 0.16$ & $2.17 \pm 0.17$ & $0.97 \pm 0.13$ & $1.01 \pm 0.11$ & $0.78 \pm 0.13$ & $0.74 \pm 0.09$ \\
\hline \multicolumn{9}{|c|}{ Gastro-esophageal electrode-balloon catheter-derived parameters } \\
\hline EMGdi,rms\%max & $11.2 \pm 2.2$ & $10.5 \pm 1.3$ & $20.5 \pm 2.6$ & $25.0 \pm 2.6$ & $58.0 \pm 4.1$ & $63.2 \pm 4.6$ & $67.0 \pm 4.9$ & $77.2 \pm 3.6$ \\
\hline Pes, tidal $\left(\mathrm{cmH}_{2} \mathrm{O}\right)$ & $5.2 \pm 0.5$ & $5.2 \pm 0.5$ & $11.2 \pm 0.9$ & $12.6 \pm 1.0$ & $28.6 \pm 2.0$ & $32.8 \pm 1.3^{*}$ & $40.0 \pm 2.9$ & $37.6 \pm 1.5$ \\
\hline Peak inspiratory Pes $\left(\mathrm{cmH}_{2} \mathrm{O}\right)$ & $-11.4 \pm 1.0$ & $-10.1 \pm 0.9$ & $-14.7 \pm 1.2$ & $-13.1 \pm 1.4$ & $-25.7 \pm 1.8$ & $-23.9 \pm 1.3$ & $-29.8 \pm 1.8$ & $-24.7 \pm 1.3^{*}$ \\
\hline Peak expiratory Pes $\left(\mathrm{cmH}_{2} \mathrm{O}\right)$ & $-6.2 \pm 0.6$ & $-4.9 \pm 0.6$ & $-3.4 \pm 0.7$ & $-0.5 \pm 1.1^{*}$ & $3.0 \pm 1.0$ & $8.9 \pm 0.9$ & $10.2 \pm 1.8$ & $13.0 \pm 1.2$ \\
\hline Pdi,tidal $\left(\mathrm{cmH}_{2} \mathrm{O}\right)$ & $10.0 \pm 1.0$ & $6.6 \pm 0.7 *$ & $10.2 \pm 2.0$ & $13.0 \pm 1.0$ & $22.4 \pm 1.3$ & $27.2 \pm 1.1^{*}$ & $26.9 \pm 1.5$ & $29.4 \pm 1.6$ \\
\hline Peak inspiratory Pdi $\left(\mathrm{cmH}_{2} \mathrm{O}\right)$ & $23.1 \pm 1.2$ & $20.8 \pm 2.4$ & $26.6 \pm 1.8$ & $24.8 \pm 2.6$ & $34.4 \pm 1.7$ & $35.1 \pm 2.3$ & $38.5 \pm 1.9$ & $35.2 \pm 2.5$ \\
\hline Peak expiratory Pdi $\left(\mathrm{cmH}_{2} \mathrm{O}\right)$ & $13.1 \pm 0.8$ & $14.2 \pm 2.4$ & $13.1 \pm 1.0$ & $11.8 \pm 2.3$ & $12.0 \pm 0.9$ & $7.9 \pm 1.9$ & $11.6 \pm 0.9$ & $5.8 \pm 1.7^{*}$ \\
\hline Peak expiratory $\mathrm{Pga}\left(\mathrm{cmH}_{2} \mathrm{O}\right)$ & $12.1 \pm 0.8$ & $11.5 \pm 2.3$ & $13.7 \pm 1.0$ & $14.8 \pm 1.8$ & $16.9 \pm 1.1$ & $22.5 \pm 1.9^{*}$ & $23.1 \pm 1.7$ & $23.6 \pm 1.9$ \\
\hline \multicolumn{9}{|l|}{ Symptom parameters } \\
\hline Dyspnea intensity (Borg 0-10 scale units) & $0.1 \pm 0.1$ & $0.0 \pm 0.0$ & $0.7 \pm 0.2$ & $0.5 \pm 0.2$ & $5.6 \pm 0.7$ & $5.5 \pm 0.5$ & $7.8 \pm 0.6$ & $7.7 \pm 0.5$ \\
\hline Dyspnea unpleasantness (Borg 0-10 scale units) & $0.1 \pm 0.1$ & $0.0 \pm 0.0$ & $0.4 \pm 0.1$ & $0.1 \pm 0.1 *$ & $4.4 \pm 0.8$ & $4.5 \pm 0.6$ & $6.2 \pm 0.8$ & $6.7 \pm 0.6$ \\
\hline Leg Discomfort (Borg 0-10 scale units) & $0.0 \pm 0.0$ & $0.0 \pm 0.0$ & $0.8 \pm 0.2$ & $0.4 \pm 0.1 *$ & $6.3 \pm 0.7$ & $5.5 \pm 0.7^{*}$ & $8.6 \pm 0.6$ & $7.4 \pm 0.7^{*}$ \\
\hline
\end{tabular}

respectively; $\mathrm{P}_{\mathrm{T}} \mathrm{O}_{2}$ and $\mathrm{P}_{\mathrm{ET}} \mathrm{CO}_{2}$, end-tidal partial pressure of $\mathrm{O}_{2}$ and $\mathrm{CO}_{2}$, respectively; $\mathrm{V}_{\mathrm{E}}$, minute ventilation; $\mathrm{V}_{\mathrm{T}}$, tidal volume; $f_{\mathrm{R}}$, breathing frequency; IC, inspiratory capacity; IRV, inspiratory reserve volume; EMGdi,rms\%max, root mean square of the crural diaphragm electromyogram (EMGdi,rms) expressed as a percentage of maximal voluntary EMGdi,rms; Pes,tidal and Pdi,tidal, tidal esophageal and transdiaphragmatic pressure swings, respectively; Pga, gastric pressure. ${ }^{*} \mathrm{p} \leq 0.05$ vs. cycle at measurement time. 
632

633

634

635

636

637

638

639

640

641

642

643

644

645

646

647

648

649

650

651

652

653

654

655

656

\section{Figure Legends}

Figure 1. Metabolic, cardiovascular and gas exchange responses during symptom-limited treadmill and cycle exercise test protocols matched for increments in work rate. Values are means $\pm \mathrm{SEM} . \dot{\mathrm{V}} \mathrm{O}_{2}$ and $\dot{\mathrm{V} C O} 2$, rate of $\mathrm{O}_{2}$ consumption and $\mathrm{CO}_{2}$ production, respectively; RER, respiratory exchange ratio; $\dot{\mathrm{V}}_{\mathrm{E}} / \dot{\mathrm{V}} \mathrm{O}_{2}$ and $\dot{\mathrm{V}}_{\mathrm{E}} / \dot{\mathrm{V} C O}{ }_{2}$, ventilatory equivalents for $\mathrm{O}_{2}$ and $\mathrm{CO}_{2}$, respectively; $\mathrm{P}_{\mathrm{ET}} \mathrm{O}_{2}$ and $\mathrm{P}_{\mathrm{ET}} \mathrm{CO}_{2}$, end-tidal partial pressure of $\mathrm{O}_{2}$ and $\mathrm{CO}_{2}$, respectively. ${ }^{*} \mathrm{p} \leq 0.05$ vs. cycle at measurement time.

Figure 2. Ventilation, breathing pattern and operating lung volume responses during symptomlimited treadmill and cycle exercise test protocols matched for increments in work rate. Values are means \pm SEM. $f_{\mathrm{R}}$, breathing frequency. ${ }^{*} \mathrm{p} \leq 0.05$ vs. cycle at measurement time.

Figure 3. Gastro-esophageal electrode-balloon catheter-derived responses during symptomlimited treadmill and cycle exercise test protocols matched for increments in work rate. Values are means \pm SEM. EMGdi,rms\%max, root mean square of the crural diaphragm electromyogram (EMGdi,rms) expressed as a percentage of maximal voluntary EMGdi,rms; Pes,tidal and Pdi,tidal, tidal esophageal and transdiaphragmatic pressure swings, respectively; Pga, gastric pressure. ${ }^{*} \mathrm{p} \leq 0.05$ vs. cycle at measurement time.

Figure 4. Perceptual responses during symptom-limited treadmill and cycle exercise test protocols matched for increments in work rate. Values are means $\pm \mathrm{SEM} . \quad \mathrm{VO}_{2}$, rate of $\mathrm{O}_{2}$ consumption; EMGdi,rms\%max, root mean square of the crural diaphragm electromyogram (EMGdi,rms) expressed as a percentage of maximal voluntary EMGdi,rms; TLC, total lung capacity. ${ }^{*} p \leq 0.05$ vs. cycle at measurement time. 

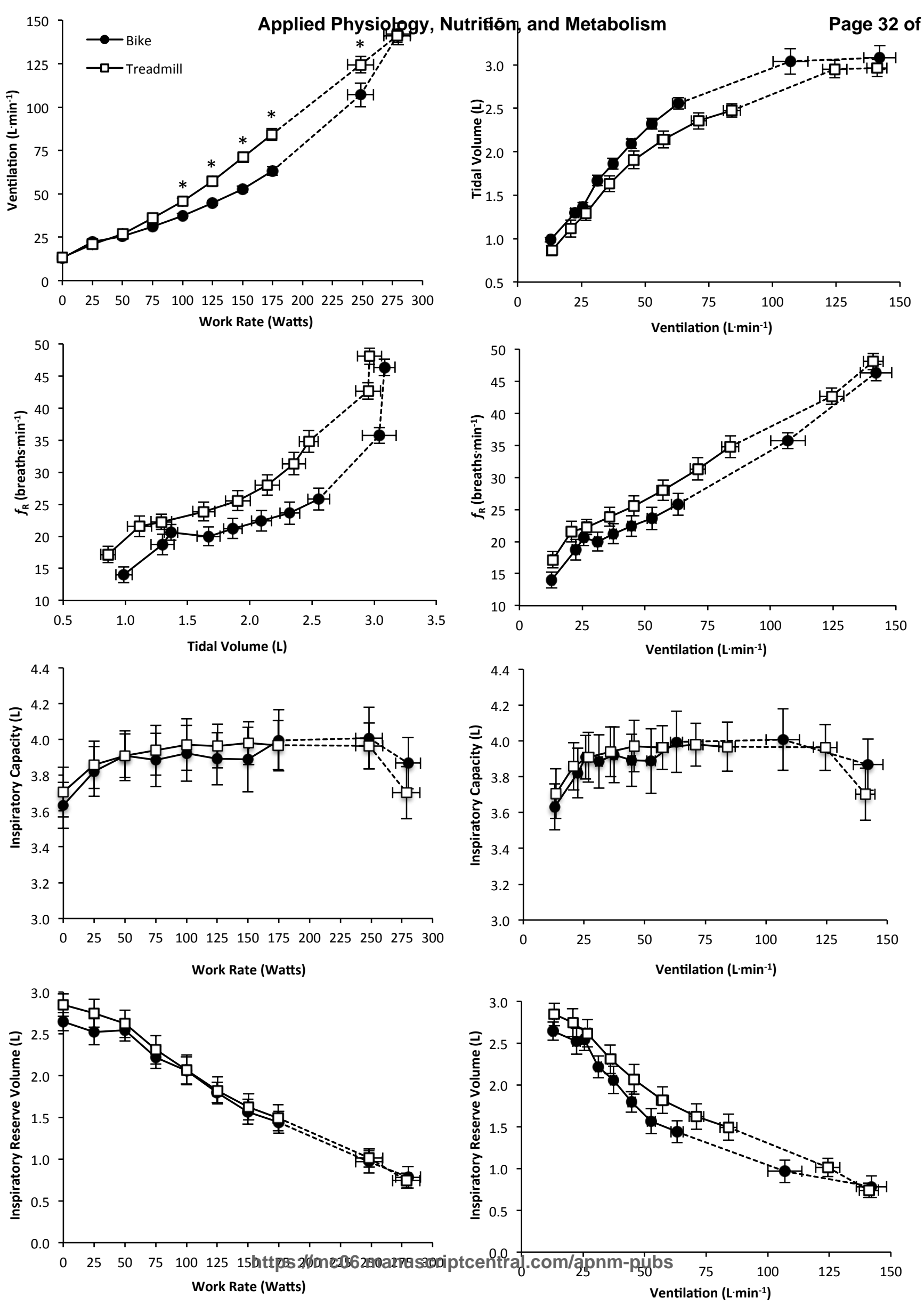

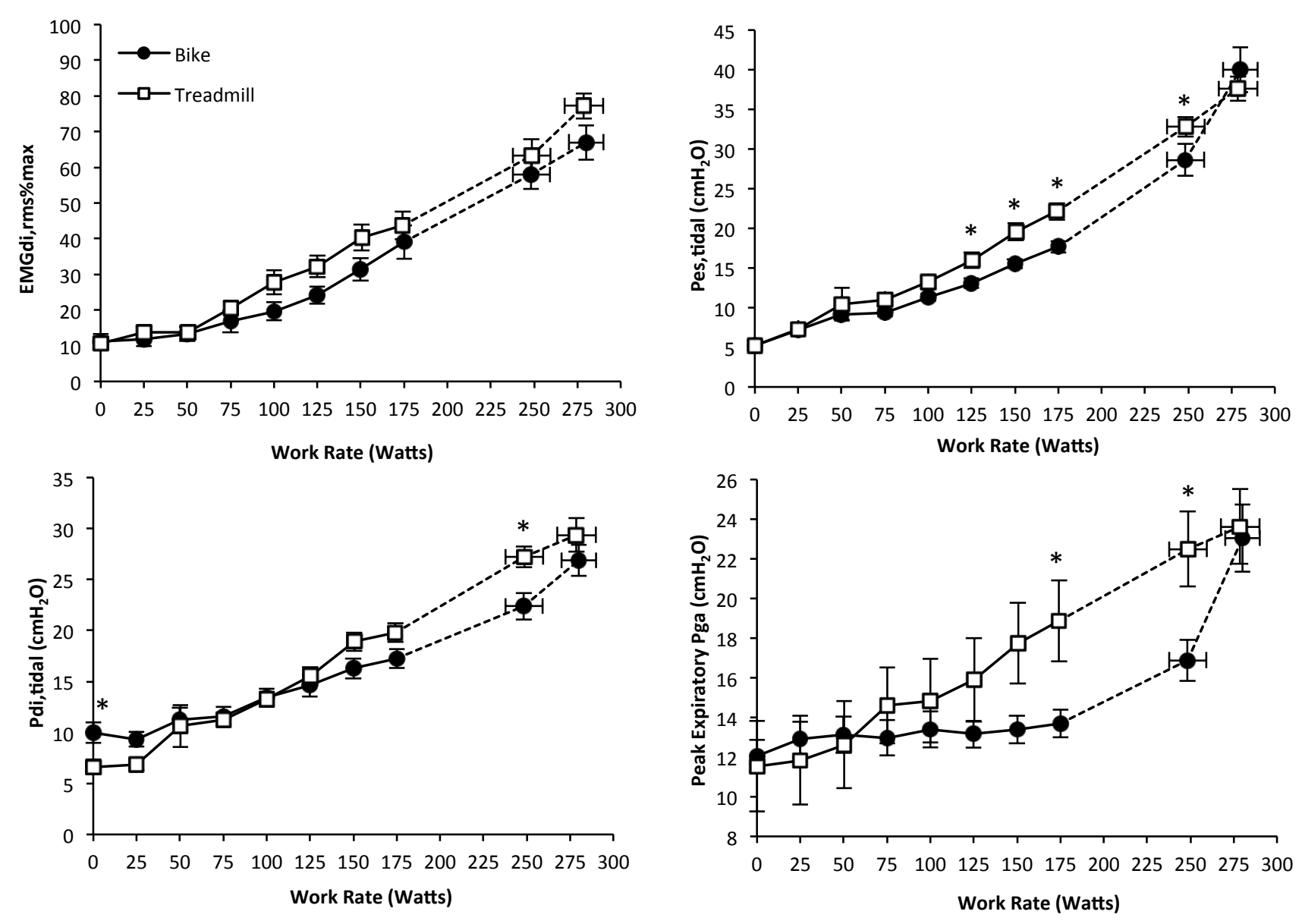

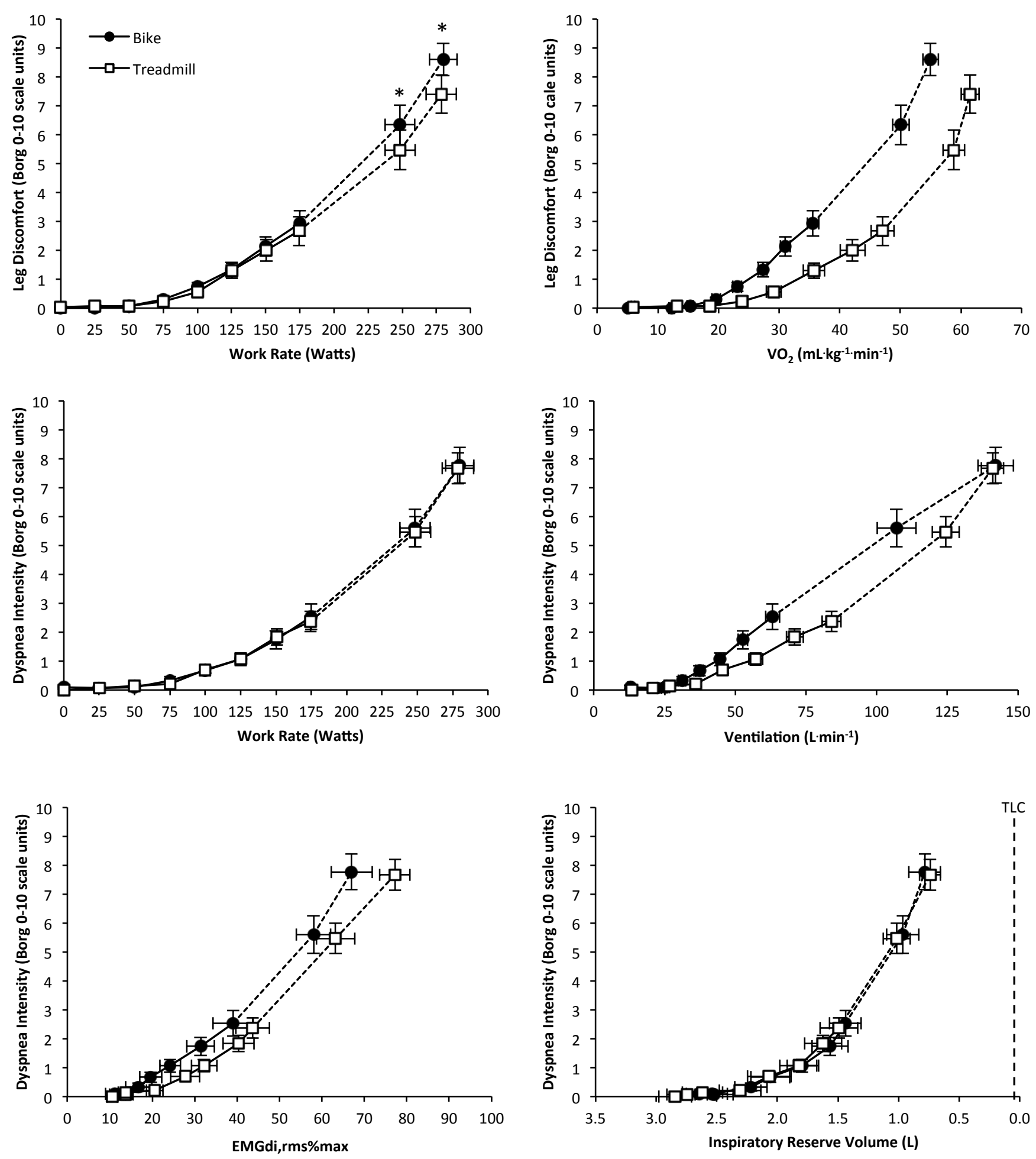